\title{
Introduction to Relativistic Collisions
}

\author{
Frank W. K. Firk \\ The Henry Koerner Center for Emeritus Faculty, Yale University \\ New Haven CT 06520
}

Abstract: This introduction is intended for students taking a first course in Nuclear and Particle Physics. Electron-positron annihilation-in-flight is discussed as a topical example of an exact relativistic analysis.

\section{4-momentum and the energy-momentum invariant}

In Classical Mechanics, the concept of momentum is important because of its rôle as an invariant in an isolated system. We therefore introduce the concept of 4-momentum in Relativistic Mechanics in order to find possible Lorentz invariants involving this new quantity. The contravariant 4-momentum is defined as:

$$
\mathrm{P}^{\mu}=\mathrm{mV} \mathrm{V}^{\mu}
$$

where $\mathrm{m}$ is the mass of the particle (it is a Lorentz scalar - the mass measured in the rest frame of the particle), and $V^{\mu}\left[\gamma c, \gamma \mathbf{V}_{N}\right]$ is the 4velocity. Here, $\gamma$ is the relativistic factor and $\mathbf{v}_{\mathrm{N}}$ is the Newtonian 3velocity,

Now, $\mathrm{V}^{\mu} \mathrm{V}_{\mu}=\mathrm{c}^{2}$, where $\mathrm{c}$ is the invariant speed of light, therefore

$$
\mathrm{P}^{\mu} \mathrm{P}_{\mu}=(\mathrm{mc})^{2}
$$

The 4-momentum can be written,

$$
\mathrm{P}^{\mu}=\left[\mathrm{m} \gamma \mathrm{c}, \mathrm{m} \gamma \mathbf{v}_{\mathrm{N}}\right]
$$


therefore,

$$
\mathrm{P}^{\mu} \mathrm{P}_{\mu}=(\mathrm{m} \gamma \mathrm{c})^{2}-\left(\mathrm{m} \gamma \mathbf{v}_{\mathrm{N}}\right)^{2}
$$

Writing

$$
\begin{aligned}
M & =\gamma \mathrm{m} \text {, the relativistic mass, we obtain } \\
\mathrm{P}^{\mu} \mathrm{P}_{\mu} & =(M c)^{2}-\left(M v_{N}\right)^{2}=(\mathrm{mc})^{2} .
\end{aligned}
$$

Multiplying throughout by $\mathrm{c}^{2}$ gives

$$
M^{2} c^{4}-M^{2} v_{N}^{2} c^{2}=m^{2} c^{4}
$$

The quantity $\mathrm{Mc}^{2}$ has dimensions of energy; we therefore write

$$
\mathrm{E}=\mathrm{Mc}^{2} \text {, }
$$

the total energy of a freely moving particle.

This leads to the fundamental invariant of dynamics

$$
\mathbf{c}^{2} \mathrm{P}^{\mu} \mathrm{P}_{\mu}=\mathrm{E}^{2}-(\mathbf{p c})^{2}=\mathrm{E}^{\mathrm{o}}
$$

where

$$
\mathrm{E}^{\mathrm{o}}=\mathrm{mc}^{2} \text { is the rest energy of the particle, and } \mathbf{p} \text { is its relativistic 3- }
$$

momentum.

The total energy can be written:

$$
E=\gamma E^{o}=E^{o}+T,
$$

where

$$
\mathrm{T}=\mathrm{E}^{\mathrm{o}}(\gamma-1)
$$

the relativistic kinetic energy. 
The magnitude of the 4-momentum is a Lorentz invariant

$$
\left|\mathrm{P}^{\mu}\right|=\mathrm{mc}
$$

The 4- momentum transforms as follows:

$$
\mathrm{P}^{\prime \mu}=\mathbf{L} \mathbf{P}^{\mu} \text {, where } \mathbf{L} \text { is the Lorentz operator. }
$$

\section{The relativistic Doppler shift}

For relative motion along the $\mathrm{x}$-axis, the equation $\mathrm{P}^{\prime \mu}=\mathbf{L} \mathbf{P}^{\mu}$ is equivalent to the equations (here, $\beta=\mathrm{v} / \mathrm{c}$ )

$$
E^{\prime}=\gamma E-\beta \gamma c p^{x}
$$

and,

$$
c p^{\prime x}=-\beta \gamma E+\gamma c p^{x}
$$

Using the Planck-Einstein equations $\mathrm{E}=\mathrm{h} v$ and $\mathrm{E}=\mathrm{p}^{\mathrm{x}} \mathrm{c}$ for photons, the energy equation becomes

$$
\begin{aligned}
v^{\prime} & =\gamma v-\beta \gamma \nu \\
& =\gamma v(1-\beta) \\
& =v(1-\beta) /\left(1-\beta^{2}\right)^{1 / 2} \\
& =v\{(1-\beta) /(1+\beta)\}^{1 / 2}
\end{aligned}
$$

This is the relativistic Doppler shift for the frequency $v^{\prime}$, measured in an inertial frame (primed) in terms of the frequency $v$ measured in another inertial frame (unprimed). 


\section{Relativistic collisions and the conservation of 4-momentum}

Consider the interaction between two particles, 1 and 2, to form two particles, 3 and 4 . ( 3 and 4 are not necessarily the same as 1 and 2). The contravariant 4-momenta are $\mathrm{P}_{\mathrm{i}}^{\mu}$ :

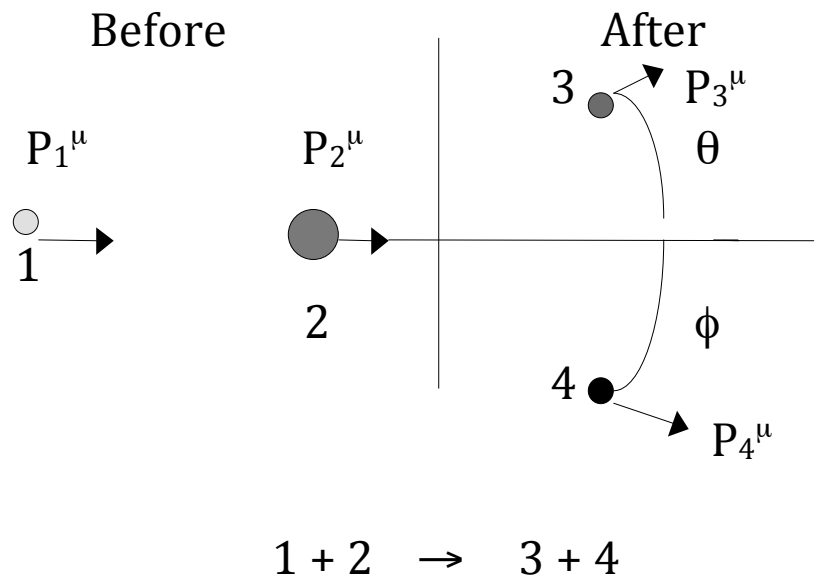

All experiments are consistent with the fact that the 4-momentum of the system is conserved. We have, for the contravariant 4momentum vectors of the interacting particles.

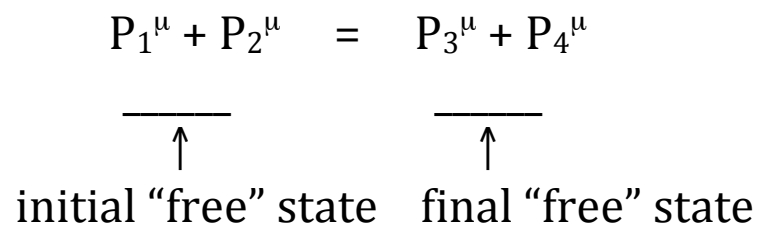

and a similar equation for the covariant 4-momentum vectors,

$$
\mathrm{P}_{1_{\mu}}+\mathrm{P}_{2_{\mu}}=\mathrm{P}_{3_{\mu}}+\mathrm{P}_{4_{\mu}}
$$

If we are interested in the change $\mathrm{P}_{1}{ }^{\mu} \rightarrow \mathrm{P}_{3}{ }^{\mu}$, then we require

$$
\mathrm{P}_{1}^{\mu}-\mathrm{P}_{3}^{\mu}=\mathrm{P}_{4}^{\mu}-\mathrm{P}_{2}^{\mu}
$$


and

$$
\mathrm{P}_{1_{\mu}}-\mathrm{P}_{3_{\mu}}=\mathrm{P}_{4_{\mu}}-\mathrm{P}_{2_{\mu}}
$$

Forming the invariant scalar products, and using $\mathrm{P}_{\mathrm{i}_{\mu}} \mathrm{P}_{\mathrm{i}}{ }^{\mu}=\left(\mathrm{E}_{\mathrm{i}}{ }^{0} / \mathrm{c}\right)^{2}$, we obtain

$$
\begin{aligned}
& \left(\mathrm{E}_{1}{ }^{0} / \mathrm{c}\right)^{2}-2\left(\mathrm{E}_{1} \mathrm{E}_{3} / \mathrm{c}^{2}-\mathbf{p}_{1} \cdot \mathbf{p}_{3}\right)+\left(\mathrm{E}_{3}{ }^{0} / \mathrm{c}\right)^{2} \\
= & \left(\mathrm{E}_{4}{ }^{0} / \mathrm{c}\right)^{2}-2\left(\mathrm{E}_{2} \mathrm{E}_{4} / \mathrm{c}^{2}-\mathbf{p}_{2} \cdot \mathbf{p}_{4}\right)+\left(\mathrm{E}_{2}{ }^{0} / \mathrm{c}\right)^{2} .
\end{aligned}
$$

Introducing the scattering angles, $\theta$ and $\phi$, this equation becomes

$$
\begin{aligned}
& E_{1} 0^{2}-2\left(E_{1} E_{3}-c^{2} p_{1} p_{3} \cos \theta\right)+E_{3}{ }^{22} \\
= & E_{2}{ }^{02}-2\left(E_{2} E_{4}-c^{2} p_{2} p_{4} \cos \phi\right)+E_{4}{ }^{02} .
\end{aligned}
$$

If we choose a reference frame in which particle 2 is at rest (the LAB frame), then $\mathbf{p}_{2}=0$ and $\mathrm{E}_{2}=\mathrm{E}_{2}{ }^{0}$, so that

$$
E_{1} 02-2\left(E_{1} E_{3}-c^{2} p_{1} p_{3} \cos \theta\right)+E_{3} 0^{2}=E_{2}{ }^{2}-2 E_{2}{ }^{0} E_{4}+E_{4} 02
$$

The total energy of the system is conserved, therefore

$$
E_{1}+E_{2}=E_{3}+E_{4}=E_{1}+E_{2} 0
$$

or

$$
\mathrm{E}_{4}=\mathrm{E}_{1}+\mathrm{E}_{2}^{0}-\mathrm{E}_{3}
$$

Eliminating $\mathrm{E}_{4}$ from the above "scalar product" equation gives

$E_{1}^{02}-2\left(E_{1} E_{3}-c^{2} p_{1} p_{3} \cos \theta\right)+E_{3}^{02}=E_{4}^{02}-E_{2}^{02}-2 E_{2}^{0}\left(E_{1}-E_{3}\right)$. 
This is the basic equation for all interactions in which two relativistic entities in the initial state interact to give two relativistic entities in the final state. It applies equally well to interactions that involve massive and massless entities.

\subsection{The Compton effect}

The general method discussed in the previous section can be used to provide an exact analysis of Compton's famous experiment in which the scattering of a photon by a stationary, free electron was studied. In this example, we have

$$
E_{1}=E_{p h} \text { (the incident photon energy), } E_{2}=E_{e}{ }^{0} \text { (the rest energy of }
$$
the stationary electron, the "target"), $E_{3}=E_{p h}$ ( the energy of the scattered photon), and $\mathrm{E}_{4}=\mathrm{E}_{\mathrm{e}}{ }^{\prime}$ (the energy of the recoilling electron). The "rest energy" of the photon is zero:

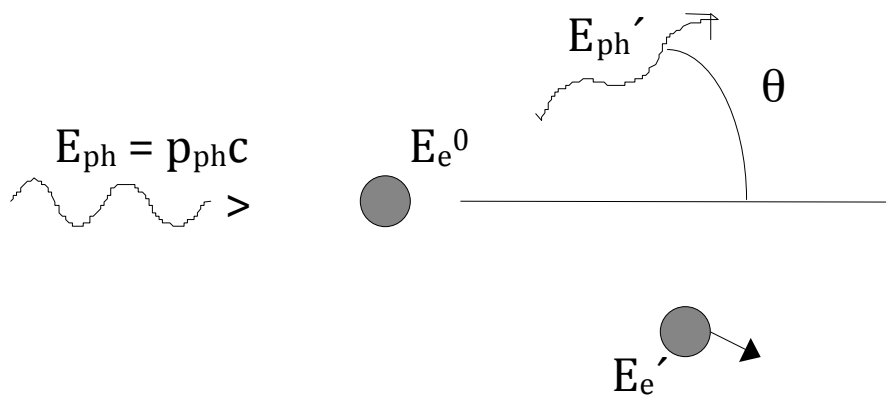

The general equation is now

$$
0-2\left(E_{p h} E_{p h}{ }^{\prime}-E_{p h} E_{p h}{ }^{\prime} \cos \theta\right)=E_{e^{02}}-2 E_{e}^{0}\left(E_{p h}+E_{e^{0}}-E_{p h}{ }^{\prime}\right)+E_{e^{02}}
$$

or 


$$
-2 E_{p h} E_{p h}{ }^{\prime}(1-\cos \theta)=-2 E_{e}^{0}\left(E_{p h}-E_{p h}{ }^{\prime}\right)
$$

so that

$$
E_{p h}-E_{p h}{ }^{\prime}=E_{p h} E_{p h}(1-\cos \theta) / E_{e}{ }^{0} .
$$

Compton measured the energy-loss of the photon on scattering and its $\cos \theta$-dependence.

\section{Relativistic inelastic collisions}

We shall consider an inelastic collision between a particle 1 and a particle 2 (initially at rest) to form a composite particle 3 . In such a collision, the 4-momentum is conserved (as it is in an elastic collision) however, the kinetic energy is not conserved. Part of the kinetic energy of particle 1 is transformed into excitation energy of the composite particle 3. This excitation energy can take many forms - heat energy, rotational energy, and the excitation of quantum states at the microscopic level. The inelastic collision is as shown:

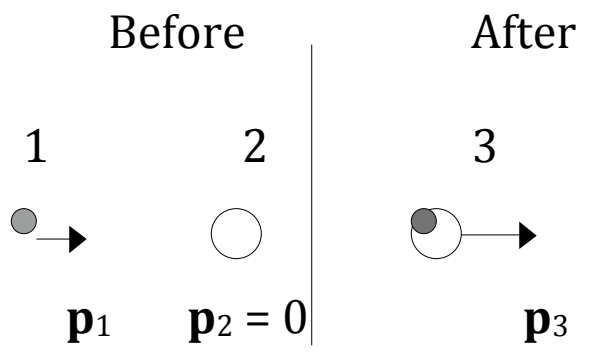

Rest energy: $\quad \mathrm{E}_{1}{ }^{0} \quad \mathrm{E}_{2}{ }^{0} \quad \mathrm{E}_{3}{ }^{0}$

Total energy: $\mathrm{E}_{1} \quad \mathrm{E}_{2}=\mathrm{E}_{2}{ }^{0} \quad \mathrm{E}_{3}$

3-momentum: $\mathbf{p}_{1} \quad \mathbf{p}_{2}=0 \quad \mathbf{p}_{3}$

Kinetic energy: $\mathrm{T}_{1} \quad \mathrm{~T}_{2}=0 \quad \mathrm{~T}_{3}$ 
In this problem, we shall use the energy-momentum invariants associated with each particle, directly:

i) $\mathrm{E}_{1}{ }^{2}-\left(\mathbf{p}_{1} \mathrm{c}\right)^{2}=\mathrm{E}_{1} 0^{2}$

ii) $\mathrm{E}_{2}{ }^{2} \quad=\mathrm{E}_{2}{ }^{\mathrm{O}} 2$

iii) $\mathrm{E}_{3}{ }^{2}-\left(\mathbf{p}_{3} \mathrm{c}\right)^{2}=\mathrm{E}_{3}{ }^{02}$.

The total energy is conserved, therefore

$$
\mathrm{E}_{1}+\mathrm{E}_{2}=\mathrm{E}_{3}=\mathrm{E}_{1}+\mathrm{E}_{2}{ }^{0} .
$$

Introducing the kinetic energies of the particles, we have

$$
\left(\mathrm{T}_{1}+\mathrm{E}_{1}^{0}\right)+\mathrm{E}_{2}{ }^{0}=\mathrm{T}_{3}+\mathrm{E}_{3}{ }^{0} .
$$

The 3-momentum is conserved, therefore

$$
\mathbf{p}_{1}+0=\mathbf{p}_{3} .
$$

Using

$$
\mathrm{E}_{3} 0^{02}=\mathrm{E}_{3}{ }^{2}-\left(\mathbf{p}_{3} \mathrm{c}\right)^{2},
$$

we obtain

$$
\begin{aligned}
E_{3} 02 & =\left(E_{1}+E_{2}\right)^{2}-\left(\mathbf{p}_{3} c\right)^{2} \\
& =E_{1}^{2}+2 E_{1} E_{2}{ }^{0}+E_{2} 0^{2}-\left(\mathbf{p}_{1} c\right)^{2} \\
& =E_{1} 0^{2}+2 E_{1} E_{2}{ }^{0}+E_{2}{ }^{2} \\
& =E_{1} 0^{2}+E_{2} 0^{0}+2\left(T_{1}+E_{1}{ }^{0}\right) E_{2}{ }^{0}
\end{aligned}
$$

or 


$$
\mathrm{E}_{3} 0^{02}=\left(\mathrm{E}_{1}^{0}+\mathrm{E}_{2}^{0}\right)^{2}+2 \mathrm{~T}_{1} \mathrm{E}_{2}^{0} \quad\left(\mathrm{E}_{3}^{0}>\mathrm{E}_{1}^{0}+\mathrm{E}_{2}^{0}\right) .
$$

Using $T_{1}=\gamma_{1} E_{1}{ }^{0}-E_{1}{ }^{0}$, where $\gamma_{1}=\left(1-\beta_{1}^{2}\right)^{-1 / 2}$ and $\beta_{1}=v_{1} / c$, we have

$$
\mathrm{E}_{3} 0^{02}=\mathrm{E}_{1} 0^{2}+\mathrm{E}_{2}{ }^{02}+2 \gamma_{1} \mathrm{E}_{1}{ }^{0} \mathrm{E}_{2}{ }^{0} .
$$

If two identical particles make a completely inelastic collision then

$$
\mathrm{E}_{3} 02=2\left(\gamma_{1}+1\right) \mathrm{E}_{1} 02
$$

\section{The Mandelstam variables}

In discussions of relativistic interactions it is often useful to introduce additional Lorentz invariants that are known as Mandelstam variables. They are, for the special case of two particles in the initial and final states $(1+2 \rightarrow 3+4)$ :

$$
\mathrm{S}=\left(\mathrm{P}_{1}^{\mu}+\mathrm{P}_{2}^{\mu}\right)\left[\mathrm{P}_{1_{\mu}}+\mathrm{P}_{2_{\mu}}\right] \text {, the total 4-momentum }
$$

invariant

$$
\begin{aligned}
& =\left(\left(E_{1}+E_{2}\right) / c,\left(p_{1}+p_{2}\right)\right)\left[\left(E_{1}+E_{2}\right) / c,-\left(p_{1}+\mathbf{p}_{2}\right)\right] \\
& =\left(E_{1}+E_{2}\right)^{2} / c^{2}-\left(p_{1}+p_{2}\right)^{2} \\
& \rightarrow \text { Lorentz invariant, } \\
t & =\left(P_{1}^{\mu}-P_{3}^{\mu}\right)\left[P_{1_{\mu}}-P_{3_{\mu}}\right], \text { the 4-momentum transfer }
\end{aligned}
$$

$(1 \rightarrow 3)$ invariant

$$
\begin{aligned}
& =\left(E_{1}-E_{3}\right)^{2} / c^{2}-\left(\mathbf{p}_{1}-\mathbf{p}_{3}\right)^{2} \\
& \rightarrow \text { Lorentz invariant, }
\end{aligned}
$$


and

$$
\mathrm{u}=\left(\mathrm{P}_{1}^{\mu}-\mathrm{P}_{4}^{\mu}\right)\left[\mathrm{P}_{1_{\mu}}-\mathrm{P}_{4}\right] \text {, the 4-momentum transfer }
$$

$(1 \rightarrow 4)$ invariant

$$
\begin{aligned}
& =\left(E_{1}-E_{4}\right)^{2} / c^{2}-\left(\mathbf{p}_{1}-\mathbf{p}_{4}\right)^{2} \\
& \rightarrow \text { Lorentz invariant. }
\end{aligned}
$$

Now,

$$
\begin{gathered}
\mathrm{sc}^{2}=\mathrm{E}_{1}{ }^{2}+2 \mathrm{E}_{1} \mathrm{E}_{2}+\mathrm{E}_{2}{ }^{2}-\left(\mathrm{p}_{1}{ }^{2}+2 \mathbf{p}_{1} \cdot \mathbf{p}_{2}+\mathrm{p}_{2}{ }^{2}\right) \mathrm{c}^{2} \\
=\mathrm{E}_{1} 0^{2}+\mathrm{E}_{2}{ }^{2}{ }^{2}+2 \mathrm{E}_{1} \mathrm{E}_{2}-2 \mathbf{p}_{1} \cdot \mathbf{p}_{2} \mathrm{c}^{2} \\
=\mathrm{E}_{1} 0^{2}+\mathrm{E}_{2}{ }^{02}+2\left(\mathrm{E}_{1}, \mathbf{p}_{1} \mathrm{c}\right)\left[\mathrm{E}_{2},-\mathbf{p}_{2} \mathrm{c}\right] . \\
\frac{\uparrow}{\text { Lorentz invariant }}
\end{gathered}
$$

The Mandelstam variable $s c^{2}$ has the same value in all inertial frames. We therefore evaluate it in the LAB frame, defined by the vectors

$$
\left[\mathrm{E}_{1}^{\mathrm{L}}, \mathbf{p}_{1}^{\mathrm{L}} \mathrm{C}\right] \text { and }\left[\mathrm{E}_{2}{ }^{\mathrm{L}}=\mathrm{E}_{2}{ }^{0},-\mathbf{p}_{2}{ }^{\mathrm{L}} \mathrm{c}=0\right]
$$

so that

$$
2\left(\mathrm{E}_{1} \mathrm{~L}_{2} \mathrm{~L}^{\mathrm{L}}-\mathbf{p}_{1}{ }^{\mathrm{L}} \cdot \mathbf{p}_{2}{ }^{\mathrm{L}} \mathbf{c}^{2}\right)=2 \mathrm{E}_{1}{ }^{\mathrm{L}} \mathrm{E}_{2}{ }^{0},
$$

and

$$
\mathrm{SC}^{2}=\mathrm{E}_{1} 0^{2}+\mathrm{E}_{2}{ }^{0} 2+2 \mathrm{E}_{1}^{\mathrm{L}} \mathrm{E}_{2}{ }^{0}
$$


We can evaluate $\mathrm{sc}^{2}$ in the center-of -mass (CM) frame, defined by the condition

$$
\begin{aligned}
\mathbf{p}_{1}{ }^{\mathrm{CM}}+\mathbf{p}_{2}{ }^{\mathrm{CM}}=0 \text { (the total 3-momentum is zero): } \\
\mathrm{sc}^{2}=\left(\mathrm{E}_{1} \mathrm{CM}^{\mathrm{M}}+\mathrm{E}_{2}{ }^{\mathrm{CM}}\right)^{2} .
\end{aligned}
$$

This is the square of the total CM energy of the system.

\subsection{The total CM energy and the production of new particles}

The quantity $c \sqrt{s}$ is the energy available for the production of new particles, or for exciting the internal structure of particles. We can now obtain the relation between the total CM energy and the LAB energy of the incident particle (1) and the target (2), as follows:

$$
\mathrm{sc}^{2}=\mathrm{E}_{1} \mathrm{O}^{2}+\mathrm{E}_{2} \mathrm{0}^{2}+2 \mathrm{E}_{1} \mathrm{E}_{2}{ }^{0}=\left(\mathrm{E}_{1}^{\mathrm{CM}}+\mathrm{E}_{2}{ }^{\mathrm{CM}}\right)^{2}=\mathrm{W}^{2} \text {, say. }
$$

Here, we have evaluated the left-hand side in the LAB frame, and the right-hand side in the CM frame!

At very high energies, $c \sqrt{S}>E_{1}{ }^{0}$ and $E_{2}{ }^{0}$, the rest energies of the particles in the initial state, in which case,

$$
\mathrm{W}^{2}=\mathrm{sc}^{2} \approx 2 \mathrm{E}_{2}{ }^{\mathrm{L}} \mathrm{E}_{2}{ }^{0} .
$$

The total CM energy, $\mathrm{W}$, available for the production of new particles therefore depends on the square root of the incident laboratory energy. This result led to the development of colliding, or intersecting, beams of particles (such as protons and anti-protons) in order to produce 
sufficient energy to generate particles with rest masses typically 100 times the rest mass of the proton $\left(\sim 10^{9} \mathrm{eV}\right)$.

\section{Positron-electron annihilation-in-flight}

A discussion of the annihilation-in-flight of a relativistic positron and a stationary electron provides a topical example of the use of relativistic conservation laws. This process, in which two photons are spontaneously generated, has been used as a source of nearly monoenergetic high-energy photons for the study of nuclear photodisintegration since 1960. The general result for a $1+2 \rightarrow 3+4$ interaction, given in section 3, provides the basis for an exact calculation of this process; we have

$$
E_{1}=E_{p o s} \text { (the incident positron energy), } E_{2}=E_{e}{ }^{0} \text { (the rest energy of }
$$
the stationary electron), $E_{3}=E_{\mathrm{ph} 1}$ (the energy of the forward-going photon), and $E_{4}=E_{p h 2}$ (the energy of the backward-going photon). The rest energies of the positron and the electron are equal. The general equation now reads

$$
E_{e}{ }^{02}-2\left\{E_{p o s} E_{p h 1}-c p_{p o s} E_{p h 1}(\cos \theta)\right\}+0=0-E_{e} e^{02}-2 E_{e}^{0}\left(E_{p o s}-E_{p h 1}\right)
$$

therefore

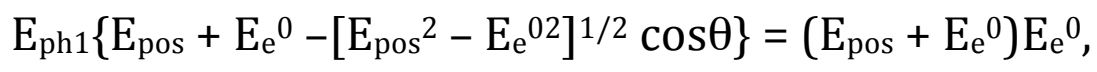

giving 


$$
\mathrm{E}_{\mathrm{ph} 1}=\mathrm{E}_{\mathrm{e}}{ }^{0} /(1-\mathrm{k} \cos \theta)
$$

where

$$
\mathrm{k}=\left[\left(\mathrm{E}_{\mathrm{pos}}-\mathrm{E}_{\mathrm{e}}^{0}\right) /\left(\mathrm{E}_{\mathrm{pos}}+\mathrm{E}_{\mathrm{e}}^{0}\right)\right]^{1 / 2} .
$$

The maximum energy of the photon, $E_{\mathrm{ph} 1}{ }^{\max }$ occurs when $\theta=0$, corresponding to motion in the forward direction; its energy is

$$
\mathrm{E}_{\mathrm{ph} 1} \max =\mathrm{E}_{\mathrm{oe}} /(1-\mathrm{k})
$$

If, for example, the incident total positron energy is $30 \mathrm{MeV}$, and $\mathrm{E}_{\mathrm{e}}^{0}=0.511 \mathrm{MeV}$ then

$$
\begin{aligned}
\mathrm{E}_{\mathrm{ph} 1} \max & =0.511 /\left[1-(29.489 / 30.511)^{1 / 2}\right] \mathrm{MeV} \\
& =30.25 \mathrm{MeV} .
\end{aligned}
$$

The forward-going photon has energy equal to the kinetic energy of the incident positron $\left(\mathrm{T}_{1}=30-0.511 \mathrm{MeV}\right)$ plus about three-quarters of the total rest energy of the positron-electron pair $\left(2 \mathrm{E}_{\mathrm{e}}{ }^{0}=1.02 \mathrm{MeV}\right)$. Using the conservation of the total energy of the system, we see that the energy of the backward-going photon is about $0.25 \mathrm{MeV}$. The method of positron-electron annihilation-in-flight provides one of the very few ways of generating nearly monoenergetic photons at high energies. The method was first proposed by Tzara (1957). 


\section{References}

The following references place the subject of relativistic collisions in the general context of Special Relativity, and provide many specific examples of the application of the theory to experiments.

1. G. Stephenson and C. W. Kilmister, Special Relativity for Physicists, Dover Publications, Inc, New York (1987).

2. Wolfgang Rindler, Introduction to Special Relativity, $2^{\text {nd }}$ edition, Clarendon Press, Oxford (1991).

3. C. Tzara, Compt. Rend. 245, 56 (1957).

4. C. Schuhl and C. Tzara, Nucl. Instr. Meth. 10, 217 (1961).

5. A. M. Baldin, V. I. Goldanskii and I. L. Rozenthal, Kinematics of Nuclear Reactions, Oxford, (1961).

6. C. F. Powell, P. H. Fowler and P. H. Perkins, The Study of Elementary Particles by the Photographic Method, Pergamon Press, Oxford (1959). 